\title{
Caracterización químico nutricional de tres semillas de oleaginosas disponibles en la región Amazonas
}

\section{Nutritional chemical characterization of three oilseeds available in the Amazon region}

\author{
Katerin Goñas y Wilmer Bernal ${ }^{1}$
}

\section{RESUMEN}

El objetivo del presente trabajo fue la caracterización de tres semillas de oleaginosas de la región Amazonas. Los siguientes parámetros evaluados fueron: humedad $(\mathrm{H})$, proteína cruda (nitrógeno total) $(\% \mathrm{PT})$, cenizas $(\mathrm{CZA} \%)$, fibra cruda $(\mathrm{FC})$, extracto etéreo (EE), extracto libre de nitrógeno (ELN). Además se determinó energía bruta (EB), fibra detergente acida (FDA), fibra detergente neutra (FDN\%), porcentaje de calcio (Ca) y fosforo (P) y digestibilidad in vitro. Las semillas de oleaginosas fueron semilla de Sacha Inchi (Plukenetia volúbilis linneo) semilla de Higuerilla (Ricinus communis) y semilla de Chía (salvia hispánica). La clasificación final de dichos insumos se realizó por la concentración de nutrientes en proteicos, energéticos y fibrosos. Concluyendo que en la región Amazonas las semillas de oleaginosas poseen un alto potencial nutricional para la alimentación animal. Sin embargo, es necesario la caracterización del perfil de aminoácidos y componentes anti nutricionales en los insumos identificados.

Palabras claves: Subproductos agroindustriales, análisis proximal, oleaginosas, valor nutricional, digestibilidad in vitro.

\begin{abstract}
The objective of this work was the characterization of three oilseeds of the Amazon region. The following parameters were evaluated: humidity $(\mathrm{H})$, crude protein (total nitrogen) $(\% \mathrm{PT})$, ash (CZA \%), crude fibre (FC), ether extract (EE), nitrogen-free extract (ELN). In addition, gross energy (EB), acid detergent fibre (FDA), neutral detergent fibre (NDF \%), calcium percentage $(\mathrm{Ca})$ and phosphorus $(\mathrm{P})$ and in vitro digestibility were determined. The seeds of oilseeds were Sacha Inchi (Plukenetia volúbilis linneo) seed, Higuerilla (Ricinus communis) seed and Chia (salvia hispánica). The final classification of these inputs was made by the concentration of nutrients in protein, energy and fibrous. Concluding that in the Amazon region. Oilseeds have a high nutritional potential for animal feed. However, it is necessary to characterize the profile of amino acids and anti-nutritional components in the identified inputs.
\end{abstract}

Keywords: Agroindustrial byproducts; proximal analysis; oleaginous; Nutritional value; In vitro digestibility.

\footnotetext{
${ }^{1}$ Universidad Nacional Toribio Rodríguez De Mendoza De Amazonas, Facultad De Ingeniería Zootecnista, Agronegocios Y Biotecnología, Escuela Profesional De Ingeniería Zootecnista. Correo: katygovilca@gmail.com
} 


\section{INTRODUCCIÓN}

En la actualidad el incremento de la población mundial y la creciente demanda alimentaria, han obligado a las empresas a aumentar el volumen de producción y consigo el incremento de subproductos agroindustriales. Es por ello que la industria alimentaria, persiguiendo su desarrollo sostenible, está aplicando cada vez más medidas para la mejora de su impacto ambiental, entre los que se encuentra la valorización y aprovechamiento de subproductos. Así mismo, este aprovechamiento crea nuevas fuentes de riqueza y de empleo que aportan mayor rentabilidad económica al proceso (Fernandez, 2014).

Los subproductos son insumos alimenticios secundarios, generalmente útiles $\mathrm{y}$ comercializables, derivados de un proceso de manufactura o reacción química, que no es el producto primario o el servicio que se produce (Osorio-Villazon \& Rojas-Vilardi, 2010). La utilización de estos como materia prima para la elaboración de concentrados se han convertido en una muy buena alternativa para minimizar los riesgos de contaminación ambiental y generar un mejor aprovechamiento de los recursos disponibles de la cadena de producción de las diferentes industrias agroalimentarias (Pacheco et al 2010).

El presente informe de investigación reporta los resultados de la composición nutricional de tres semillas de oleaginosas, recursos potenciales para ser utilizados en la alimentación animal de interés zootécnico. Los análisis que se determinaron fueron: análisis proximal además FDA, FDN, Ca, $P$ y pruebas de digestibilidad in vitro.

\section{MATERIALES Y MÉTODOS}

La población estuvo conformada por tres oleaginosas más representativas de la región Amazonas.

\section{Lugar de Estudio}

La caracterización nutricional se realizó en el laboratorio de Nutrición Animal y Bromatología de los Alimentos del Instituto de Investigación en Ganadería y Biotecnología (IGBI), ubicado en el campus universitario de la Universidad Nacional Toribio Rodríguez de Mendoza, Chachapoyas, Amazonas, Perú.

\section{Caracterización nutricional}

Se caracterizó la semilla de Sacha Inchi (Plukenetia volúbilis linneo), semilla de Higuerilla (Ricinus communis) y semilla de Chía (salvia hispánica). Se determinaron los siguientes parámetros según los métodos de la AOAC: H (método 925.09), PC (método 928.08), CZA (método 942.05), FC (método 962.09), EE (método 920.39), ELN (método 923.03), EB (bomba calorimétrica). El poder calórico se determinó con un calorímetro isoperibólico (PARR Calorimeter., USA). También se determinó FDA (método 937.19), FDN (método 937.18), Ca y P (método 927.02) y digestibilidad in vitro (protocolo establecido en el digestor in vitro DAISY II, USA).

\section{Obtención y preparación de muestras}

Las muestras fueron tomadas de diferentes provincias de la región Amazonas; Chachapoyas (Chachapoyas, El Molino), Luya (Luya, Trita), Bongará (Pedro Ruiz).

Para la preparación de las muestras se usó de 2 a 3 $\mathrm{kg}$ de muestras, las mismas que fueron envasadas en bolsas y rotuladas con código, lugar de 
procedencia y nombre del propietario. Se determinó la altitud sobre el nivel del mar y coordenadas del lugar de producción con el uso de un GPS (GARMMIN, México). Luego, se trasladaron las muestras hasta el Laboratorio de Nutrición Animal y Bromatología de Alimentos de la UNTRM, para su preparación y análisis.

Las muestras fueron pre-secadas en estufa (Ecocell, USA) a $65^{\circ} \mathrm{C}$ por un periodo de 10 a 12 horas. Las muestras secadas parcialmente, fueron molidas en un molino de laboratorio con cribas de $3 \mathrm{~mm}$. Después, $200 \mathrm{~g}$ de muestras fueron almacenadas en frascos con tapa rosca para luego determinar los diferentes componentes nutricionales según los protocolos mencionados previamente.

\section{Análisis estadístico}

Se evaluaron las características nutricionales y de digestibilidad de oleaginosas con un análisis estadístico descriptivo, con cinco repeticiones $(\mathrm{n}=5)$ por cada sub producto. Se empleó la prueba de promedios de Tukey $(\alpha=0.05)$. Los datos fueron procesados $\mathrm{y}$ analizados mediante el programa estadístico Statistix V.8.

\section{RESULTADOS}

La caracterización nutricional de oleaginosas disponibles en la región Amazonas, constituye una necesidad importante para su inclusión como fuente alimenticia en la producción animal. Los insumos evaluados se describen en la Tabla 1.

Tabla 1. Promedio y digestibilidad de Oleaginosas.

\begin{tabular}{llll}
\hline Componentes & Sacha Inchi & Higuerilla & Chía \\
\hline CZA \% & $3.17 \pm 0,3^{\text {a }}$ & $5.37 \pm 0,6^{\text {a }}$ & $4.09 \pm 1,2^{\text {a }}$ \\
EB (Mcal $/ \mathrm{kg})$ & $7.10 \pm 0,2^{\mathrm{a}}$ & $6.84 \pm 0,2^{\mathrm{a}}$ & $6.10 \pm 1,1^{\mathrm{b}}$ \\
EE \% & $39.19 \pm 6,7^{\mathrm{a}}$ & $34.55 \pm 5,3^{\mathrm{a}}$ & $37.34 \pm 2,1^{\mathrm{a}}$ \\
ELN \% & $17.27 \pm 0,8^{\mathrm{a}}$ & $15.57 \pm 0,6^{\mathrm{a}}$ & $13.67 \pm 0,7^{\mathrm{a}}$
\end{tabular}

\begin{tabular}{llll} 
FC \% & $8.99 \pm 5,9^{\mathrm{b}}$ & $32.78 \pm 6,4^{\mathrm{a}}$ & $20.2 \pm 4,1^{\mathrm{ab}}$ \\
FDA \% & $44.68 \pm 2,1^{\mathrm{a}}$ & $37.05 \pm 5,1^{\mathrm{a}}$ & $58.82 \pm 6,6^{\mathrm{a}}$ \\
FDN \% & $64.34 \pm 0,1^{\mathrm{ab}}$ & $48.03 \pm 1,2^{\mathrm{b}}$ & $73.21 \pm 0,5^{\mathrm{a}}$ \\
H \% & $2.62 \pm 0,3^{\mathrm{b}}$ & $4.46 \pm 0,6^{\mathrm{ab}}$ & $4.76 \pm 1,3^{\mathrm{a}}$ \\
PT \% & $28.75 \pm 0,1^{\mathrm{a}}$ & $18.96 \pm 0,2^{\mathrm{b}}$ & $19.94 \pm 0,0^{\mathrm{b}}$ \\
Ca \% & $0.21 \pm 0,0^{\mathrm{a}}$ & $0.35 \pm 0,0^{\mathrm{a}}$ & $0.52 \pm 0,0^{\mathrm{a}}$ \\
P \% & $0.5 \pm 0,0^{\mathrm{a}}$ & $0.52 \pm 0,2^{\mathrm{a}}$ & $0.51 \pm 0,2^{\mathrm{a}}$ \\
Digestibilidad & $55.34 \pm 7,8^{\mathrm{a}}$ & $63.22 \pm 1,8^{\mathrm{a}}$ & $21.79 \pm 2,9^{\mathrm{b}}$ \\
\hline
\end{tabular}

En la Tabla se observa los \% de energía bruta (EB), fibra cruda (FC) y proteínas totales (PT). Letras diferentes en la fila a, b y c muestran diferencia significativa $(\mathrm{p}<0.05)$. Elaboración propia.

El porcentaje de proteína en las oleaginosas fue de $28.75 \pm 0.07,19.94 \pm 0,0$ y $18.96 \pm 0,2$ en sacha inchi, chía e higuerilla; respectivamente, siendo la semilla de sacha inchi es el insumo con más porcentaje de proteína.

Se observa que el porcentaje de energía bruta en la semilla de sacha inchi, higuerilla y chía fue de $7.10 \pm 0,2,6.84 \pm 0.2$ y $6.10 \pm 1.1$ respectivamente.

El porcentaje de fibra cruda de las oleaginosas fue de $32.78 \pm 6.4,20.22 \pm 4.1$ y $8.22 \pm 5.9$ en higuerilla, chía y sacha inchi; respectivamente, siendo la semilla con mayor porcentaje de fibra la semilla de higuerilla.

En porcentaje de digestibilidad se obtuvo valores de $63.22 \pm 10.8,55.34 \pm 7.8$ y $21.79 \pm 2.9$, en higuerilla, sacha inchi y chía; respectivamente, siendo la más digestible la semilla de higuerilla.

\section{DISCUSIÓN}

Hurtado (2013), mostró un contenido de proteínas, grasas y digestibilidad in vitro en semilla de sacha inchi de $29.85 \%, \quad 42.57 \%$ y $67.62 \%$; respectivamente, acercándose a nuestros valores encontrados de $28.75 \% \pm 0.1, \quad 39.19 \% \pm 6.7 \quad \mathrm{y}$ $55.34 \% \pm 7.8$ de proteínas, extracto etéreo $\mathrm{y}$ 
digestibilidad in vitro; respectivamente, esta cercanía en valores puede deberse al método usado para la determinación de los análisis nutricionales.

Duarte et al. (2008), indican que la torta de higuerilla puede ser usada en la dieta de ovejas como un alimento rico en proteína, recomendando hasta un $8 \%$ en la ración total sin que cause reducción en el consumo y digestibilidad, Deus de O y Tavares (2011) reportaron un 32\% de proteína en torta de higuerilla difiriendo con nuestro resultado que fue de $18.96 \% \pm 0.2$ esto puede deberse a la variedad de la semilla y los factores medio ambientales en las que se desarrolló la planta, además de la forma de presentación $\mathrm{y}$ disponibilidad de nutrientes.

Ayerza y Cortes (2011), determinaron un rango para la determinación de proteína en semilla de chía, este resultado fue entre 19 y $26 \%$ estando nuestros resultados entre el rango establecido con $19.94 \% \pm 0.0$. Jiménez, (2013) indica que en la actualidad la semilla de chía es de gran interés gracias a su alto contenido de ácidos grasos poliinsaturados, fibra, proteína y antioxidantes y que puede ser considerado como insumo para la alimentación animal.

\section{CONCLUSIONES}

La semilla de sacha inchi es la semilla que sobresale entre las oleaginosas, por presentar mayores valores nutricionales comparado a higuerilla y a chía.

\section{REFERENCIA BIBLIOGRÁFICA}

AOAC Association of Official Analytical Chemists. (2005). Official Methods icial Methods of Analysis, cap 968.28,44,17, cap. 3. 2. 05 .

Ayerza R, Coates W. (2011). Protein content, oil content and fatty acid profiles as potential criteria to determine the origin of commercially grown chia (Salvia hispanica L.). Ind Crop Prod. 2011; 34:1366-71.

Deus de O, N., \& Tavares, M. (2011). Allergens and toxins from oleaginous plants: problems and solitions, environmental impact of biofuels. Allergens and toxins from oleaginous plants: problems and solitions, environmental impact of biofuels, 29 .

Duarte, M. J., Delmondes, M. A. \& Severino, L. S. (2008). Utilização de coprodutos da mamona na alimentação animal, III. Congreso Brasilero de Mamona. Energía y ricinoquimica. Salvador.

Fernandez A. (2014) Transformación de subproductos y residuos de agroindustria de cultivos templados, subtropicales y tropicales en carne y leche bovina. Instituto nacional de tecnología agropecuaria centro regional buenos aires sur estación experimental 
agropecuaria bordenave. EEA Bordenave, 2014.

Hurtado, Z. (2013). Análisis composicional de la torta y aceite de semillas de Sacha inchi (plukenetia volubilis) cultivada en Colombia. Universidad Nacional de Colombia facultad de ciencias agropecuarias, escuela de posgrados Palmira, Colombia. p. 45.

Jiménez P., Paula; Masson S., Lilia; Quitral R., Vilma. (2013). Composición química de semillas de chía, linaza y rosa mosqueta y su aporte en ácidos grasos omega-3 Revista Chilena de Nutrición, vol. 40, núm. 2, junio, 2013, pp. 155-160 Sociedad Chilena de Nutrición, Bromatología y Toxicología Santiago, Chile

Osorio-Villazon, M.,y Rojas Vilardi,F.(2010). Introducción al proceso de subproductos. Consumer Eroski. 